\title{
STEMODIA PRATENSIS (SCROPHULARIACEAE), SOBRE SU PRESENCIA EN BOLIVIA
}

\author{
MARÍA DE LAS MERCEDES SOSA ${ }^{1}$
}

\begin{abstract}
Summary: Sosa, M. M. 2009. Stemodia pratensis (Scrophulariaceae), on its presence in Bolivia. Bonplandia 18(1): 19-23. ISSN: 0524-0476.

In this work the presence of Stemodia pratensis (Aubl.) C.P.Cowan (Scrophulariaceae) in Bolivia, is confirmed. Description, illustrations and a key to separate it of the remaining species from Bolivia, are included.
\end{abstract}

Key words: Gratiolae, taxonomy, Stemodia.

Resumen: Sosa, M. M. 2009. Stemodia pratensis (Scrophulariaceae), sobre su presencia en Bolivia. Bonplandia 18(1): 19-23. ISSN: 0524-0476.

\begin{abstract}
En este trabajo se confirma la presencia de Stemodia pratensis (Aubl.) C.P.Cowan (Scrophulariaceae) en Bolivia. Se incluyen la descripción, ilustraciones y una clave para separarla de las restantes especies de Bolivia.
\end{abstract}

Palabras clave: Gratiolae, taxonomía, Stemodia.

\section{Introducción}

Stemodia L. (Scrophulariaceae, Antirrhinoideae, Gratiolae) es un género pantropical que comprende 49 especies, de las cuales 29 viven en América (Turner \& Cowan, 1993a).

En recientes viajes de recolección efectuados en Bolivia, se ha recogido un ejemplar de Stemodia cuyas características permiten identificarla como S. pratensis (Aubl.) C.P. Cowan. Esta especie fue citada para Bolivia por Kuntze (1898), bajo el nombre de Stemodiacra foliosa Kuntze (Benth.) para la
Provincia Velasco en el Departamento de Santa Cruz, pero sin indicar ejemplar de herbario. Posteriormente Minod (1918) se basó en ese trabajo y la mencionó para Bolivia como Stemodia foliosa Benth.

Foster (1958), en el catálogo de los helechos y plantas con flores de Bolivia cita solamente a Stemodia lanceolata Benth., S. parviflora Ait. (=S. verticillata (Mill.) Hassl.) y S. stricta Cham. \& Schltdl. Por su parte, Turner \& Cowan (1993b), en su revisión de las especies sudamericanas mencionan cuatro especies para Bolivia, Stemodia ericifolia (Kuntze) K. Schum. y las tres anteriormente citadas por Foster (1958). El ejemplar recien-

${ }^{1}$ Facultad de Ciencias Exactas y Naturales y Agrimensura (UNNE), Instituto de Botánica del Nordeste, Casilla de Correo 209, 3400 Corrientes, Argentina. E-mail:mdlmsvg@yahoo.com.ar 
temente recolectado confirma su presencia y eleva a cinco el número de especies de Stemodia registradas en ese país.

En este trabajo se describe e ilustra la novedad, se aportan datos acerca de su distribución geográfica y hábitat, y se incluye una clave que permite distinguirla de las otras especies que viven en Bolivia.

\section{Resultados}

\section{Stemodia pratensis (Aubl.) C.P.Cowan}

Figs. 1, 2 y 3

Cowan, C. P., Phytologia 74 (4): 309. 1993.

Matourea pratensis Aubl., Hist. Pl. Guiane 2: 642. 1775. Typus: French Guiana, Meadows of Cayenne Island, w/o date, Aublet s. n. (holotipus P?).

Stemodia foliosa Benth., J. Bot. (Hooker) 2: 46. 1840. Typus: British Guiana, Roraima, 1842-1843. Schomburgk 513/787 (lectotypus K; isolectotypi BM, NY! foto).

Stemodiacra foliosa (Benth.) Kuntze, Revis. Gen. Pl. 2: 466, 1891.

Subfruticosa de 50 a $180 \mathrm{~cm}$ alt. Tallo moderado a densamente hirsuto con pelos multiseptados, a menudo con pelos glandulares mezclados. Hojas brevemente pecioladas, 2 a 4 por nudo, 3-8 cm long. x 1-3 cm lat., lanceoladas u ovado-lanceoladas, ápice agudo y base cuneada, margen serrado, glandulosopubescentes a hirsutas, sobre todo a lo largo de las venas, epifilo ampolloso. Flores solitarias, axilares, sin bractéolas; pedicelo 5-20 mm long., piloso o glanduloso-pubescente. Corola 5-7 mm long., lila, con el interior del tubo blanco, notablemente bilabiada, labio superior (posterior) entero emarginado, mucho más corto que el inferior (anterior) que es trilobado, con venas impresas. Interior del tubo corolino pubescente cerca de la base, la pubescencia se prolonga hacia el labio inferior. Cápsula estrechamente ovoide, semillas ovoides.

Distribución geográfica y hábitat: Esta especie se distribuye en el este de Brasil, Vene- zuela y Trinidad y Tobago, donde probablemente haya sido introducida (Turner \& Cowan, 1993b). Se encuentra en suelos arenosos húmedos o inundables y en proximidades de ríos y lagunas. El material coleccionado en Bolivia se encontró en un cerrado arbóreo abierto, cercano a una laguna, con suelo de arenas rosadas (Fig. 2).

Obs. 1: Turner \& Cowan (1993b) expresan que las formas robustas de Stemodia pratensis se asemejan superficialmente a $S$. suffruticosa Kunth, pero la distinguen porque esta última crece en el noroeste de Sudamérica (Colombia, Ecuador y Perú), entre los 1000 y 2600 m (Fig. 3). El análisis de los ejemplares permite puntualizar otras diferencias entre estas dos especies. Stemodia suffruticosa posee corola bilabiada, con ambos labios de casi igual longitud, y el interior del tubo glabro. La corola de $S$. pratensis es marcadamente bilabiada, con el labio superior mucho más corto que el inferior, y el interior del tubo es pubescente (Fig. 1 D).

Obs. 2: La combinación Stemodia pratensis, basada en Matourea pratensis, fue realizada por C. P. Cowan. Sin embargo, en el Index Kewensis (Davies, 1996) se atribuye erróneamente esta combinación a B. L. Turner.

Material estudiado: BOLIVIA. Santa Cruz: Prov. Velasco, a 23,3 km S de Santa Ana, entrada a San Rafael, 16 46'30'S, 6040'30”W. $382 \mathrm{~m}$ s.m., 23-I-2005 (fl.), Seijo \& al. 3703 (CTES, LPB, NY, SI). BRASIL. Bahia: $10 \mathrm{~km} \mathrm{~L} \mathrm{de}$ Ibotirama, Rod. BR-242, 11-X-1981 (fl, fr), Hatschbach 44152 (CTES); Serra do Tombador (Mun. Morro do Chapéu), 15-VII-1979 (fl.), Hatschbach \& Guimarães 42341 (CTES); Mun. Salvador, Campus Universitario de Ondina, UFBA, 24-I-97 (fl), Guedes 4846 (CTES); Olivença, 2-3 km N (Mun. Ilhéus), 11-IV-1992 (fl), G. Hatschbach, M. Hatschbach \& Barbosa 57008 (CTES); Mun. Valença, camino a Guaibim, a $3 \mathrm{~km}$ de la rodovia Valença-Nazaré, aprox. 13 21'S 39 03’W. ca 100 m s.m., 13-I-1997 (fl), Arbo \& al. 7150 (CTES). Mato Grosso do Sul: Fazenda Cáceres (Mun. Corumbá), 11-VI-1994 (fl, fr), Hatschbach, Pott \& Silva 60925 (CTES, K). 


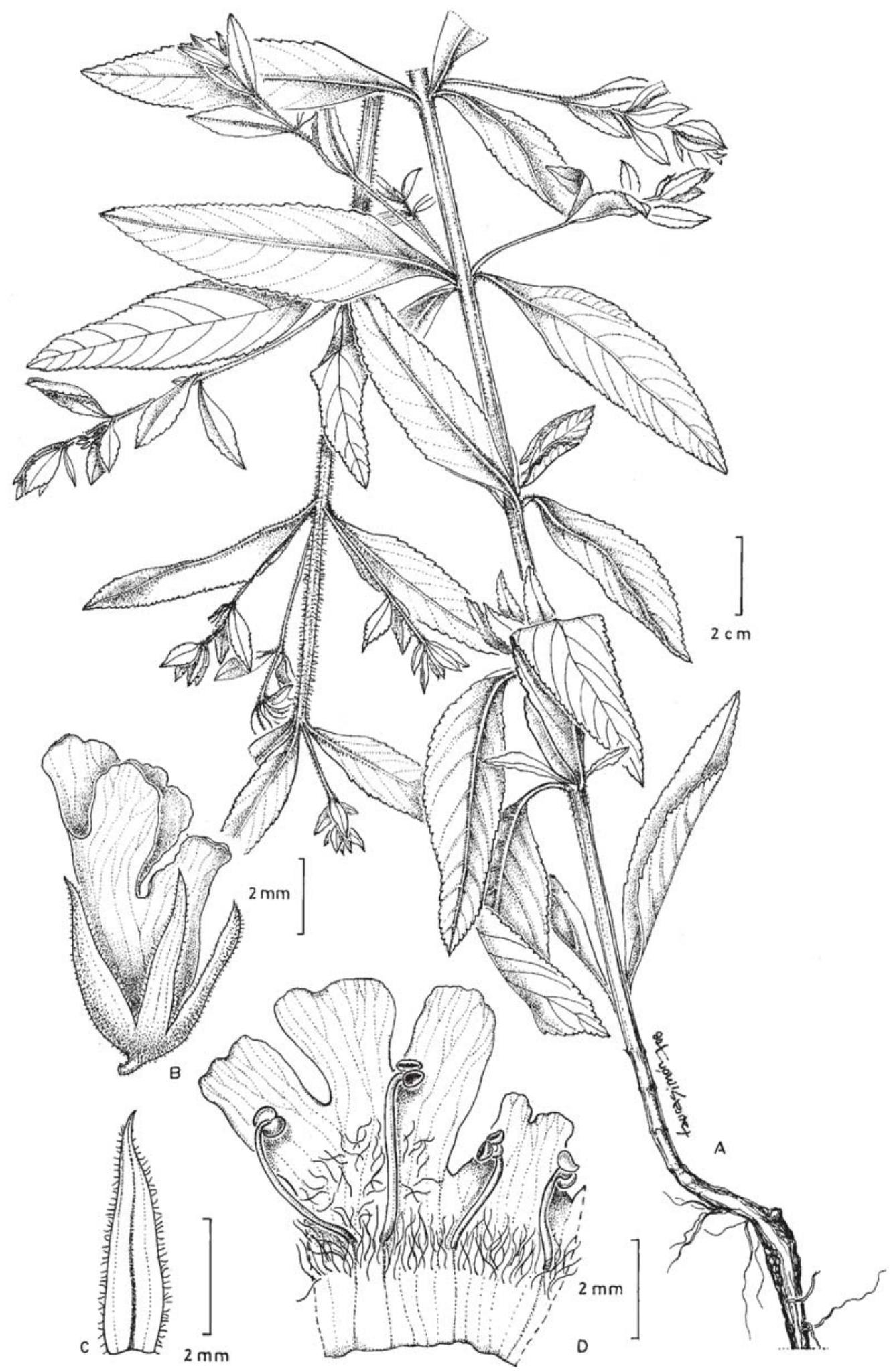

Fig. 1. Stemodia pratensis. A: planta. B: flor. C: detalle de un sépalo. D: corola abierta mostrando los tricomas y los estambres (A-D, Seijo et al. 3703). 


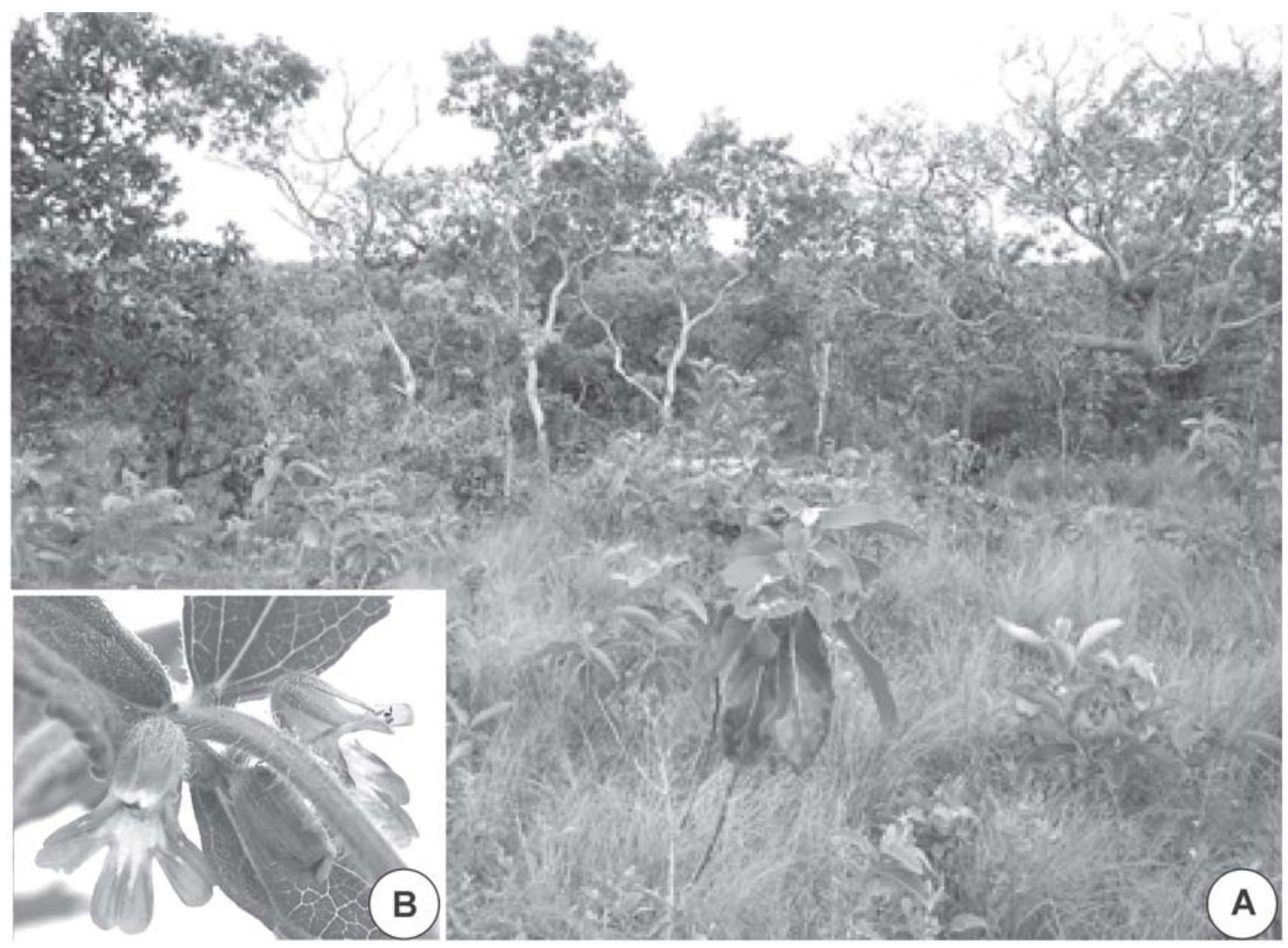

Fig. 2. A: cerrado húmedo donde crece Stemodia pratensis en Bolivia. B: detalle de una rama florífera de Stemodia pratensis.

\section{Clave para diferenciar las especies de Stemodia de Bolivia}

1. Plantas subfruticosas, erectas, hasta $180 \mathrm{~cm}$ alt.

S. pratensis (Aubl.) C.P.Cowan

1’. Plantas herbáceas, erectas, decumbentes o estoloníferas.

2. Flores ebracteoladas, axilares. Hierbas pequeñas, erectas, decumbentes o estoloníferas.

3. Hojas pecioladas, láminas ovadas.

S. verticillata (Mill.) Hassl.

3’. Hojas sésiles, lineares.

S. ericifolia (Kuntze) K. Schum.

2' Flores bibracteoladas, dispuestas en espigas. Hierbas erectas.

4. Hojas lanceoladas. Corola 12-16 mm long.

S. lanceolata Benth.

4’. Hojas ovado-lanceoladas a ovado-elípticas. Corola 5-11 mm long.

S. stricta Cham. \& Schltdl. 


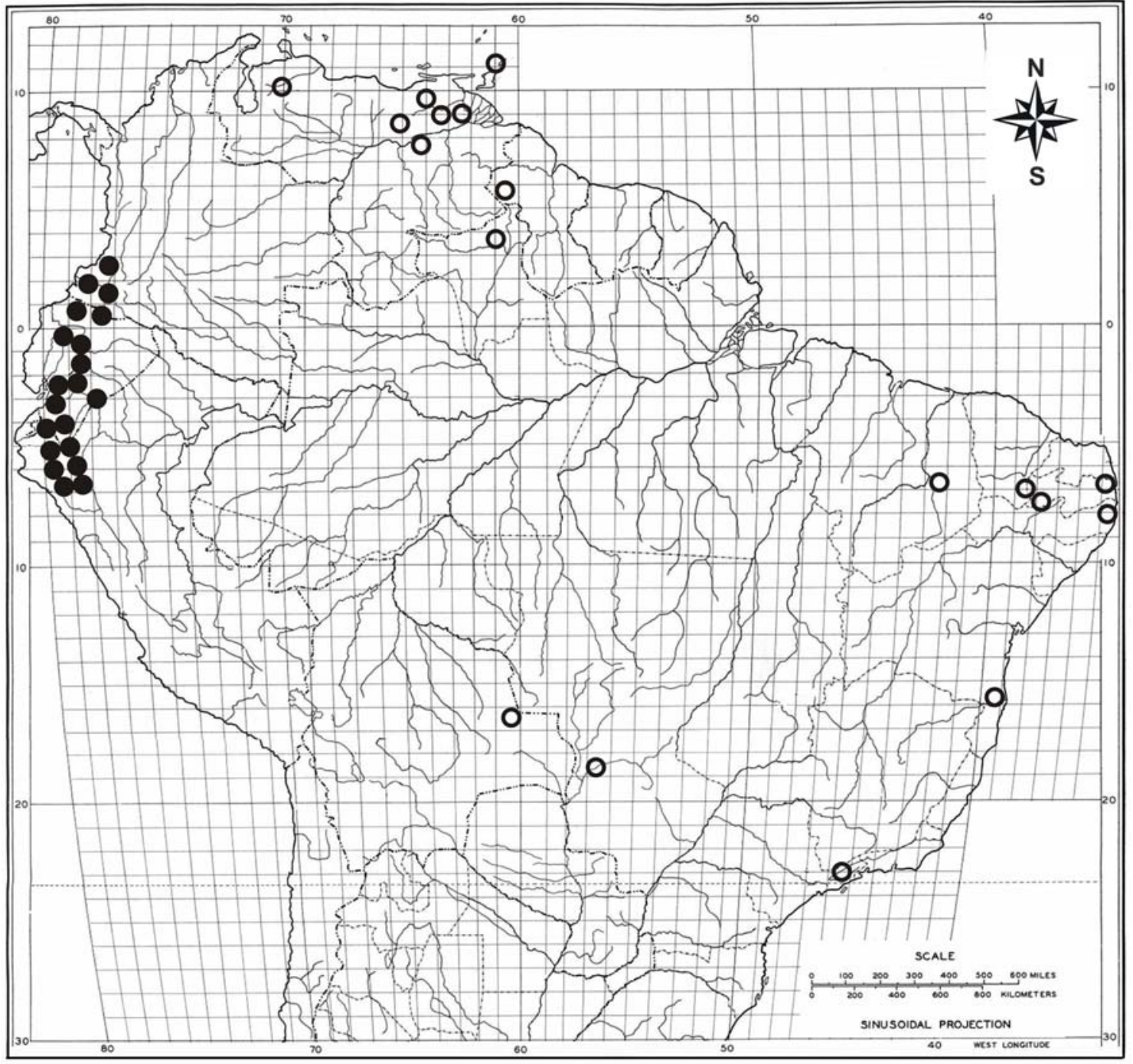

Fig. 3. Distribución geográfica de Stemodia pratensis

) y S. suffruticosa

\section{Agradecimientos}

A Guillermo Seijo y Viviana Solís Neffa por el material coleccionado y las fotografías tomadas en el campo. A Sara G. Tressens por las valiosas sugerencias aportadas. A Laura Simón por los dibujos realizados. A Massimiliano Dematteis por la lectura crítica del manuscrito.

\section{Bibliografía}

DAVIES, R. A. 1996. Index Kewensis. Suppl. 20 (19911995). 338 pp. Royal Botanical Gardens, Kew.
FOSTER, R. C. 1958. A catalogue of the ferns and flowering plants of Bolivia. Contr. Gray Herb. 184:1223.

KUNTZE, O. 1898. Revisio Generum Plantarum. Vol. 3 (2): 239.

MINOD, M. 1918. Contribution a l'étude du genre Stemodia et du groupe des Stemodiées en Amèrique. Thèse $N^{\circ}$ 606. Université de Genève, Genève.

TURNER, B. L. \& C. C. [P.] COWAN. 1993a. Taxonomic overview of Stemodia (Scrophulariaceae) for North America and the West Indies. Phytologia 74 (2): 61-103.

- 1993b. Taxonomic overview of Stemodia (Scrophulariaceae) from South America. Phytologia 74 (4): 281-324.

Original recibido el 9 de febrero de 2009; aceptado el 15 de junio de 2009. 
BONPLANDIA 18(1). 2009 